\title{
Synthesis and Surface Chemistry of 2D TiVC
}

\section{Solid-Solution MXenes}

Sanaz Yazdanparast ${ }^{1 *}$, Sina Soltanmohammad ${ }^{1}$, Annika Fash-White ${ }^{2}$, Garritt J. Tucker ${ }^{2}$, and Geoff L. Brennecka ${ }^{1}$

${ }^{1}$ Department of Metallurgical and Materials Engineering, Colorado School of Mines, Golden, CO 80401, USA

${ }^{2}$ Department of Mechanical Engineering, Colorado School of Mines, Golden, CO 80401, USA

*E-mail: syazdanparast@mines.edu 


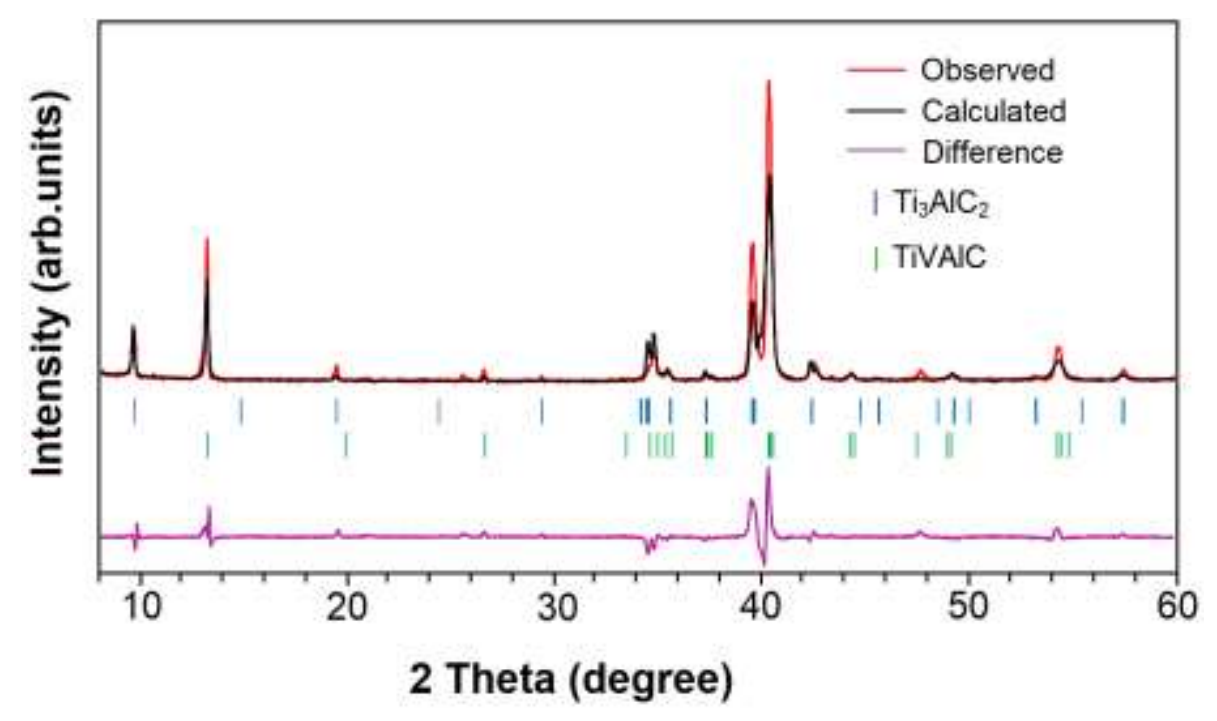

Figure S1. Rietveld refined XRD pattern of as-synthesized MAX phase.

XRD and SEM of $\operatorname{TiVCT}_{x}-\mathrm{HF}$
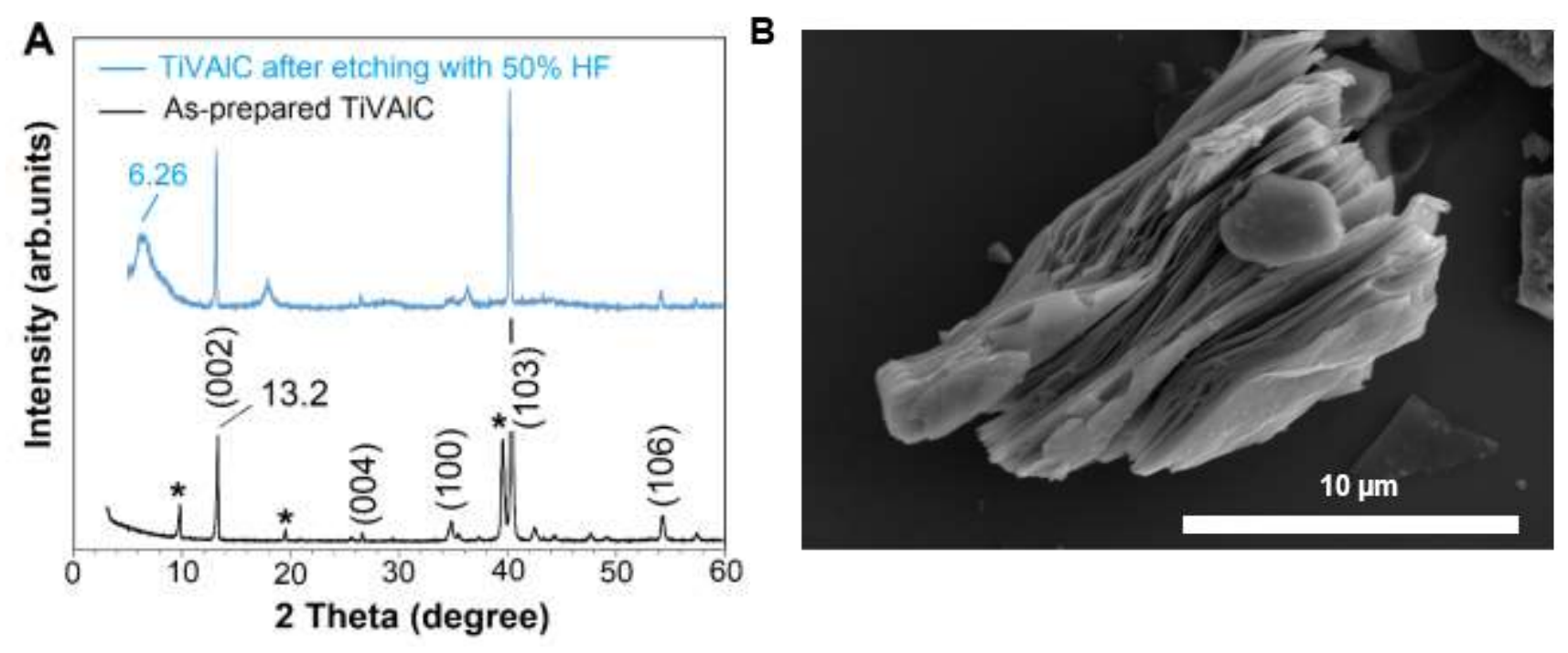

Figure S2. A) XRD $\left(\mathrm{CuK}_{\alpha}\right)$ patterns of as-prepared TiVAlC MAX phase and after etching with $50 \% \mathrm{HF}$ for $24 \mathrm{~h}$ at room temperature. Appearance of a new peak at $2 \Theta=6.26^{\circ}$ shows formation of MXene with an interlaying spacing of $1.41 \mathrm{~nm}$. B) SEM image of the stacked TiVCT ${ }_{x}$ MXene synthesized by $50 \% \mathrm{HF}$ for $24 \mathrm{~h}$ at room temperature. 


\section{DFT calculations}
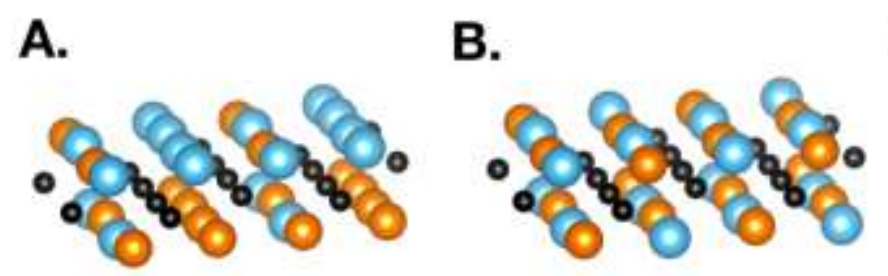

C.

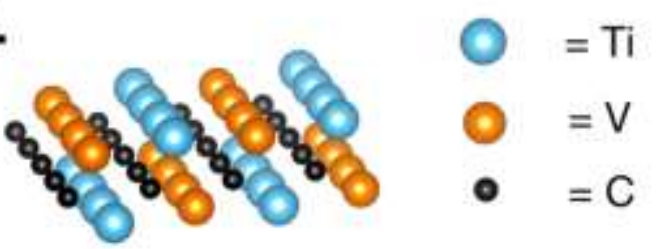

D.
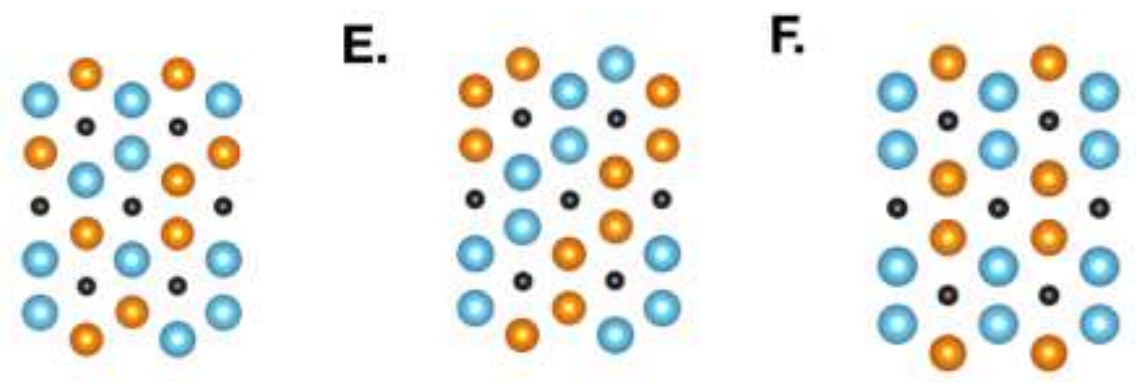

Figure S3. Three base structures representing solid solution TiVC MXene. A-C) Side views and D-F) top views of the different configurations used in the DFT calculations.

Table S1. Calculated cohesive energy for three TiVC base structures.

\begin{tabular}{|l|l|l|l|l|}
\hline Structure & $\begin{array}{l}\mathrm{TiVCO}_{2} \\
(\mathrm{eV} / \text { atom })\end{array}$ & $\begin{array}{l}\mathrm{TiVCF}_{2} \\
(\mathrm{eV} / \text { atom })\end{array}$ & $\begin{array}{l}\mathrm{TiVC}(\mathrm{OH})_{2} \\
(\mathrm{eV} / \text { /atom })\end{array}$ & $\begin{array}{l}\mathrm{TiVCCl}_{2} \\
(\mathrm{eV} / \text { /atom })\end{array}$ \\
\hline $\mathrm{A}$ & -8.4027 & -7.3640 & -6.9312 & -6.5683 \\
\hline $\mathrm{B}$ & -8.3946 & -7.3746 & -6.9397 & -6.5762 \\
\hline $\mathrm{C}$ & -8.4175 & -7.3629 & -6.9297 & -6.5658 \\
\hline Average & -8.4049 & -7.3672 & -6.9335 & -6.5701 \\
\hline
\end{tabular}

Table S2. Calculated adsorption energy for three TiVC base structures.

\begin{tabular}{|l|l|l|l|l|}
\hline Structure & $\begin{array}{l}\mathrm{TiVCO}_{2} \\
(\mathrm{eV} / \text { atom })\end{array}$ & $\begin{array}{l}\mathrm{TiVCF}_{2} \\
(\mathrm{eV} / \text { atom })\end{array}$ & $\begin{array}{l}\mathrm{TiVC}(\mathrm{OH})_{2} \\
(\mathrm{eV} / \text { atom })\end{array}$ & $\begin{array}{l}\mathrm{TiVCCl}_{2} \\
(\mathrm{eV} / \text { atom })\end{array}$ \\
\hline A & -9.3894 & -6.7925 & -5.3369 & -4.8033 \\
\hline B & -9.3727 & -6.8228 & -5.3700 & -4.8268 \\
\hline C & -9.4193 & -6.7828 & -5.3246 & -4.7901 \\
\hline Average & -9.3938 & -6.7994 & -5.3438 & -4.8067 \\
\hline
\end{tabular}




\section{XPS Analysis}

The atomic concentrations were calculated by the actual area of the peaks and atomic sensitivity factors of XPS.

Table S3. XPS fitting results for TiVCTx MXene prepared by 50\% HF.

\begin{tabular}{|c|c|c|c|c|}
\hline Region & $\overline{B E}(\mathrm{eV})$ & $\begin{array}{l}\text { FWHM } \\
(\mathrm{eV})\end{array}$ & $\begin{array}{l}\% \text { Atomic } \\
\text { concentration }\end{array}$ & Assigned to \\
\hline $\mathrm{Ti} 2 \mathrm{p}_{3 / 2}$ & $\begin{array}{l}454.6 \\
455.8 \\
458.2\end{array}$ & $\begin{array}{l}0.6 \\
2.1 \\
2\end{array}$ & $\begin{array}{l}2.7 \\
5.2 \\
1.6\end{array}$ & $\begin{array}{l}\text { Ti-C } \\
\text { Ti-O } \\
\text { C-Ti-Fx }(\mathrm{III})^{2}\end{array}$ \\
\hline $\mathrm{V} 2 \mathrm{p}_{3 / 2}$ & $\begin{array}{l}513 \\
514 \\
515.4 \\
518.3\end{array}$ & $\begin{array}{l}0.9 \\
1.5 \\
2.7 \\
3\end{array}$ & $\begin{array}{l}0.9 \\
2.3 \\
2.9 \\
1.8\end{array}$ & $\begin{array}{l}\mathrm{V}-\mathrm{C}^{3} \\
\mathrm{~V}_{\mathrm{x}} \mathrm{O}_{\mathrm{y}}{ }^{4} \\
\mathrm{~V}_{\mathrm{z}} \mathrm{O}_{\mathrm{w}}{ }^{5} \\
\mathrm{~V}-\mathrm{F}^{6}\end{array}$ \\
\hline $\mathrm{C} 1 \mathrm{~s}$ & $\begin{array}{l}281.8 \\
282.3 \\
285 \\
286.8 \\
288.7\end{array}$ & $\begin{array}{l}0.6 \\
1.3 \\
2 \\
2 \\
1.5\end{array}$ & $\begin{array}{l}5 \\
5.5 \\
18.2 \\
8.5 \\
2.4\end{array}$ & $\begin{array}{l}\mathrm{C}-\mathrm{Ti}^{7} \\
\mathrm{C}-\mathrm{V}^{8} \\
\mathrm{C}-\mathrm{C}^{7} \\
\mathrm{CH} \mathrm{H}_{\mathrm{x}} / \mathrm{C}-\mathrm{O}^{2,7,9} \\
\mathrm{C}=\mathrm{O} \text { and/or } \mathrm{C}-\mathrm{F}^{1,7,10}\end{array}$ \\
\hline O1s & $\begin{array}{l}529.8 \\
531.9 \\
533.5 \\
535 \\
\end{array}$ & $\begin{array}{l}1.7 \\
2 \\
1.9 \\
2\end{array}$ & $\begin{array}{l}6.5 \\
9.3 \\
5.9 \\
1.2\end{array}$ & $\begin{array}{l}\mathrm{O}-\mathrm{Ti}^{2,10,11} \\
\mathrm{~V}=\mathrm{O}^{6} \\
\mathrm{OH}^{7} \\
\mathrm{H}_{2} \mathrm{O}^{12}\end{array}$ \\
\hline F1s & $\begin{array}{l}683.6 \\
685 \\
686.5 \\
688\end{array}$ & $\begin{array}{l}1.1 \\
1.8 \\
1.5 \\
1.5\end{array}$ & $\begin{array}{l}0.6 \\
14 \\
4.1 \\
1.2\end{array}$ & $\begin{array}{l}\text { F-V } \\
\text { C-Ti-F }(\mathrm{III})^{2,13} \\
\text { F-Metal-Organic }^{14} \\
\text { F-Organic }^{14}\end{array}$ \\
\hline
\end{tabular}


Table S4. XPS fitting results for TiVCTx MXene prepared by $1.9 \mathrm{M} \mathrm{LiF/} 12 \mathrm{M} \mathrm{HCl}$.

\begin{tabular}{|c|c|c|c|c|}
\hline Region & $\mathrm{BE}(\mathrm{eV})$ & $\begin{array}{l}\text { FWHM } \\
(\mathrm{eV})\end{array}$ & $\begin{array}{l}\% \text { Atomic } \\
\text { concentration }\end{array}$ & Assigned to \\
\hline Ti $2 p_{3 / 2}$ & $\begin{array}{l}453 \\
454.6 \\
457.2 \\
458 \\
459.6\end{array}$ & $\begin{array}{l}1.3 \\
1.5 \\
1.1 \\
0.7 \\
2\end{array}$ & $\begin{array}{l}0.3 \\
0.2 \\
2.9 \\
0.5 \\
0.7\end{array}$ & $\begin{array}{l}\mathrm{Ti}^{15} \\
\mathrm{Ti}^{1} \mathrm{C}^{1} \\
\mathrm{Ti}^{2}-\mathrm{O}^{2,16,17} \\
\mathrm{Ti}^{18} \mathrm{Cl}^{18} \\
\mathrm{C}_{-} \mathrm{Ti}-\mathrm{F}_{\mathrm{x}}(\mathrm{III})^{2}\end{array}$ \\
\hline $\mathrm{V} 2 \mathrm{p}_{3 / 2}$ & $\begin{array}{l}512.7 \\
514.7 \\
515.7 \\
517 \\
519.7 \\
\end{array}$ & $\begin{array}{l}2 \\
1.5 \\
1.9 \\
2.8 \\
2.6 \\
\end{array}$ & $\begin{array}{l}0.5 \\
1.2 \\
2.2 \\
1.3 \\
1.2 \\
\end{array}$ & $\begin{array}{l}\mathrm{V}-\mathrm{C}^{3} \\
\mathrm{~V}-\mathrm{Cl}^{4} \\
\mathrm{~V}_{\mathrm{x}} \mathrm{O}_{\mathrm{y}}{ }^{5} \\
\mathrm{~V}_{\mathrm{x}} \mathrm{O}_{\mathrm{y}}{ }^{5} \\
\mathrm{~V}-\mathrm{F}^{6} \\
\end{array}$ \\
\hline $\mathrm{C} 1 \mathrm{~s}$ & $\begin{array}{l}280.6 \\
283 \\
284.7 \\
286.9 \\
288.5 \\
291\end{array}$ & $\begin{array}{l}0.6 \\
1.3 \\
2 \\
2 \\
1.5 \\
1.5\end{array}$ & $\begin{array}{l}1.2 \\
12.5 \\
9.8 \\
14.2 \\
1.6 \\
0.8\end{array}$ & $\begin{array}{l}\mathrm{C}-\mathrm{Ti}^{7} \\
\mathrm{C}-\mathrm{V}^{8} \\
\mathrm{C}-\mathrm{C}^{7} \\
\mathrm{CH} / \mathrm{C}-\mathrm{O}^{2,7,9} \\
\mathrm{C}=\mathrm{O} \text { and/or } \mathrm{C}-\mathrm{F}^{1,7,10} \\
\mathrm{C}-\mathrm{F} \text { and/or } \mathrm{Cl}^{19,20}\end{array}$ \\
\hline O1s & $\begin{array}{l}528.7 \\
530 \\
532.4 \\
534.7\end{array}$ & $\begin{array}{l}1.2 \\
2.5 \\
2.5 \\
2.5\end{array}$ & $\begin{array}{l}6.7 \\
18.3 \\
7 \\
2.1\end{array}$ & $\begin{array}{l}\mathrm{O}-\mathrm{Ti}^{17,21,22} \\
\mathrm{~V}=\mathrm{O}^{6} \\
-\mathrm{OH}^{7} \\
\mathrm{H}_{2} \mathrm{O}^{7}\end{array}$ \\
\hline F1s & $\begin{array}{l}683.2 \\
684.9 \\
688.5 \\
693.1 \\
\end{array}$ & $\begin{array}{l}2.4 \\
2.1 \\
5 \\
3.1 \\
\end{array}$ & $\begin{array}{l}1.4 \\
2 \\
2.2 \\
6 \\
\end{array}$ & $\begin{array}{l}\text { F-V } \\
\text { C-Ti-Fx }(\mathrm{III})^{2,13} \\
\text { F-Metal-Organic }^{14} \\
\text { F-Organic }^{14}\end{array}$ \\
\hline $\mathrm{Cl} 2 \mathrm{p}_{3 / 2}$ & $\begin{array}{l}196.7 \\
198.5 \\
201.3 \\
\end{array}$ & $\begin{array}{l}1.7 \\
2 \\
1.2 \\
\end{array}$ & $\begin{array}{l}1.8 \\
1 \\
0.3 \\
\end{array}$ & $\begin{array}{l}\mathrm{Cl}-\mathrm{V}^{3} \\
\mathrm{Cl}-\mathrm{Ti}^{18} \\
\mathrm{Cl}-\mathrm{C}^{23}\end{array}$ \\
\hline
\end{tabular}

\section{REFERENCES}

1. Kong, F.; He, X.; Liu, Q.; Qi, X.; Zheng, Y.; Wang, R.; Bai, Y. Improving the Electrochemical Properties of MXene Ti 3 C 2 Multilayer for Li-Ion Batteries by Vacuum Calcination. Electrochim. Acta 2018, 265, 140-150.

2. Halim, J.; Cook, K. M.; Naguib, M.; Eklund, P.; Gogotsi, Y.; Rosen, J.; Barsoum, M. W. X-Ray Photoelectron Spectroscopy of Select Multi-Layered Transition Metal Carbides 
(MXenes). Appl. Surf. Sci. 2016, 362, 406-417.

3. Groenenboom, C. J.; Sawatzky, G.; de Liefde Meijer, H. J.; Jellinek, F. Electron Spectroscopy of Some Cyclopentadienylcycloheptatrienylmetal Compounds. J. Organomet. Chem. 1974, 76 (1), 6-8.

4. Horvath, B.; Strutz J.; Geyer-Lippmann J.; Horvath E.G. Preparation, Properties, and ESCA Characterization of Vanadium Surface Compounds on Silicagel.II, Z. Anorg. Allg. Chem. 1981, 483 (12), 181-192.

5. Colton, R. J.; Guzman, A. M.; Rabalais, J. W. Electrochromism in Some Thin-Film Transition-Metal Oxides Characterized by x-Ray Electron Spectroscopy. J. Appl. Phys. 1978, 49 (1), 409-416.

6. Groult, H.; Devilliers, D.; Kumagai, N.; Nakajima, T.; Matsuo, Y. Vanadium Oxide Fluoride-Graphite Intercalation Compounds: Structural Characteristics and Electrochemical Insertion of Lithium Cations. J. Electrochem. Soc. 1996, 143 (7), 2093-2099.

7. Kumar, S.; Lei, Y.; Alshareef, N. H.; Quevedo-Lopez, M. A.; Salama, K. N. Biofunctionalized Two-Dimensional Ti3C2 MXenes for Ultrasensitive Detection of Cancer Biomarker. Biosens. Bioelectron. 2018, 121 (August), 243-249.

8. Antonik, M. D.; Lad, R. J.; Christensen, T. M. Clean Surface and Oxidation Behavior of Vanadium Carbide, VC0.75(100). Surf. Interface Anal. 1996, 24 (10), 681-686.

9. Jayaweera, P. M.; Quah, E. L.; Idriss, H. Photoreaction of Ethanol on TiO2(110) SingleCrystal Surface. J. Phys. Chem. C 2007, 111 (4), 1764-1769.

10. Han, M.; Yin, X.; Wu, H.; Hou, Z.; Song, C.; Li, X.; Zhang, L.; Cheng, L. Ti3C2MXenes with Modified Surface for High-Performance Electromagnetic Absorption and Shielding in the X-Band. ACS Appl. Mater. Interfaces 2016, 8 (32), 21011-21019. 
11. Yamamoto, S.; Bluhm, H.; Andersson, K.; Ketteler, G.; Ogasawara, H.; Salmeron, M.;

Nilsson, A. In Situ X-Ray Photoelectron Spectroscopy Studies of Water on Metals and Oxides at Ambient Conditions. J. Phys. Condens. Matter 2008, 20 (18).

12. Schulze, P. D.; Shaffer, S. L.; Hance, R. L.; Utley, D. L. Adsorption of Water on Rhenium Studied by XPS. J. Vac. Sci. Technol. A Vacuum, Surfaces, Film. 1983, 1 (1), 97-99.

13. Sultana, T.; Georgiev, G. L.; Auner, G.; Newaz, G.; Herfurth, H. J.; Patwa, R. XPS Analysis of Laser Transmission Micro-Joint between Poly (Vinylidene Fluoride) and Titanium. Appl. Surf. Sci. 2008, 255 (5 PART 2), 2569-2573.

14. Huang, A.; Chen, L. H.; Chen, C. H.; Tsai, H. Y.; Tung, K. L. Carbon Dioxide Capture Using an Omniphobic Membrane for a Gas-Liquid Contacting Process. J. Memb. Sci. 2018, 556 (April), 227-237.

15. Anderson, C. R.; Lee, R. N.; Morar, J. F.; Park, R. L. Comparison of Aps and Fresca Core Level Binding Energy Measurements. J. Vac. Sci. Technol. 1981, 20 (3), 617-621.

16. Schier, V.; Halbritter, J.; Karlsruhe, K. ARXPS-Analysis of Sputtered TiC, SiC and Tio.SSio.SC Layers. J. Anal. Chem. 1993, 227-232.

17. Habib, T.; Zhao, X.; Shah, S. A.; Chen, Y.; Sun, W.; An, H.; Lutkenhaus, J. L.; Radovic, M.; Green, M. J. Oxidation Stability of Ti3C2Tx MXene Nanosheets in Solvents and Composite Films. npj 2D Mater. Appl. 2019, 3 (1), 1-6. https://doi.org/10.1038/s41699-019-0089-3.

18. Mousty-Desbuquoit, C.; Riga, J.; Verbist, J. J. Solid State Effects in the Electronic Structure of TiCl4 Studied by XPS. J. Chem. Phys. 1983, 79 (1), 26-32.

19. Kang, W.; Li, S. Preparation of Fluorinated Graphene to Study Its Gas Sensitivity. RSC Adv. 2018, 8 (41), 23459-23467.

20. Lindberg, B. J.; Hamrin, K.; Johansson, G.; Gelius, U.; Fahlman, A.; Nordling, C.; 
Siegbahn, K. Molecular Spectroscopy by Means of Esca. Phys. Scr. 1970, 1 (5-6), 286-298.

21. Cao, Y.; Deng, Q.; Liu, Z.; Shen, D.; Wang, T.; Huang, Q.; Du, S.; Jiang, N.; Lin, C. Te; Yu, J. Enhanced Thermal Properties of Poly(Vinylidene Fluoride) Composites with Ultrathin Nanosheets of MXene. RSC Adv. 2017, 7 (33), 20494-20501.

22. Dementjev, A. P.; Ivanova, O. P.; Vasilyev, L. A.; Naumkin, A. V.; Nemirovsky, D. M.; Shalaev, D. Y. Altered Layer as Sensitive Initial Chemical State Indicator*. J. Vac. Sci. Technol. A Vacuum, Surfaces, Film. 1994, 12 (2), 423-427.

23. Clark, D. T.; Kilcast, D.; Musgrave, W.K.R. Molecular core binding energies for some monosubstituted benzenes, as determined by $X$-ray photoelectron spectroscopy. J. Chem. Soc. D: Chem. Commun. 1971, 516b-518. 